\title{
Visual and Refractive Outcomes after Cataract Surgery with a Monofocal Intraocular Lens Using Two Optical Biometers: IOL Master and Aladdin with EyeCeeOne Preloaded IOL
}

\author{
Alberto Parafita-Fernandez ${ }^{1,2^{*}}$, Maria N Moraña ${ }^{1,2}$, Jorge García-Borregón ${ }^{1}$, Martin Jabif ${ }^{1}$, Joao C \\ Soares da Costa ${ }^{1}$, Alba Feal ${ }^{1}$, Eloy Viso ${ }^{1}$ \\ ${ }^{1}$ Ophthalmology Department, Complexo Hospitalario Universitario de Pontevedra, Spain \\ ${ }^{2}$ Clínica Oftalmológica Dr Parafita, Ribeira, Spain \\ *Corresponding Author: Alberto Parafita-Fernandez, Ophthalmology Department, Complexo Hospitalario \\ Universitario de Pontevedra, Spain and Clínica Oftalmológica Dr Parafita, Ribeira, Spain; \\ Email: alberto.parafita@outlook.com
}

Received Date: 16-04-2021; Accepted Date: 10-05-2021; Published Date: 17-05-2021

Copyright $^{\odot} 2021$ by Fernandez AP, et al. All rights reserved. This is an open access article distributed under the terms of the Creative Commons Attribution License, which permits unrestricted use, distribution, and reproduction in any medium, provided the original author and source are credited.

\begin{abstract}
Purpose: To report visual and refractive outcomes obtained with a preloaded, hydrophobic, monofocal, intraocular lens in two series of patients with two different optic biometers for power calculation.

Methods: Two comparable series of 38 eyes that underwent cataract surgery, followed by EyeCeeOne Preloaded IOL implantation, in two different centers from March 2018 to march 2019 are retrospectively studied. Two different optic biometers (IOLMaster and Aladdin), one in each center, were used to measure the eyes of the patients and calculate pseudophakic IOL power with SRK/T formula. Exclusion criteria included: corneal astigmatism larger than 1.5D, intraoperative complications (i.e. posterior capsular tear, vitreous loss) cases in which capsular tension ring was implanted, cases with postoperative complications unrelated to IOL placement (i.e. macular edema, endothelial failure). Statistical comparisons were made using the unpaired Student t- Test assuming heteroscedasticity.
\end{abstract}

Results: Uncorrected distance visual acuity in decimal notation was $0.87 \pm 0.13$ in Aladdin group and $0.84 \pm 0.19$ in IOLMaster group $(\mathrm{p}=0.409)$, mean spherical equivalent (SE) obtained was $-0.21 \pm 0.34$ and $-0.13 \pm 0.43(\mathrm{p}=0.359)$ and the mean difference among predicted and 
obtained SE was $-0.02 \pm 0.34$ and $0.10 \pm 0.39(\mathrm{p}=0.179)$ respectively. $84.2 \%$ and $81.5 \%$ of patients were among $\pm 0.5 \mathrm{D}$ of $\mathrm{SE}$ respectively.

Conclusion: In this study the EyeCeeOne Preloaded IOL proved to be a safe and effective IOL for pseudophakia. Predictable and satisfactory refractive outcomes can be achieved using two different biometers. Further studies with extreme axial lengths are needed to evaluate IOL performance in those situations.

\section{Keywords}

Preloaded IOL; Aladdin; IOL Master; Refractive Outcomes

\section{Introduction}

Cataract surgery is one of the most frequent surgical procedures performed worldwide. Thanks to Sir Harold Ridley, the use of Intraocular Lenses (IOLs) is the standard in our daily basis. Surgical techniques evolved from intracapsular surgery with large incisions to Small Incision Cataract Surgery (SICS), phacoemulsification with posterior chamber foldable IOLS, femtosecond assisted phacoemulsification, and a myriad of possible lenses (monofocal, bifocal, trifocal, EDOF, toric). All of these changes were adopted to reduce surgical times, achieve a quicker return to the daily activities and improve visual and refractive outcomes.

Modern microincisional cataract surgery is more than just a therapeutic surgery. It has become a refractive procedure as well, thus, cataract surgeons need to be familiarized with the several options available to achieve patient's expectations. Many patients demand spectacle independence for far, intermediate and near vision, so multifocal IOLs are a good option for them [1]. On the other hand, other patients are not so demanding in relation to spectacle independence or do not wish to deal with photic phenomena associated to presbyopia correcting IOLs, but still desire good visual acuity and to be as spectacle independent as possible. In these cases, monofocal IOLs can successfully achieve the desired refractive outcome, either targeting emmetropia in both eyes or targeting emmetropia in the dominant eye and myopia between 1.5 and -2.0 in the non-dominant one (so called monovision or combined vision) [2].

Successful outcomes rely on accurate biometry for IOL power calculation. Many devices (optical biometers) are market available for these estimations, being the IOL Master 500 the most widely used for comparison with other optical biometers (Carl Zeiss Meditec, Germany) [3-6]. When different optical biometers are used, the Lens A-Constant needs to be adjusted (optimized) for each device, as using an erroneous A-Constant can lead to a systematic error in IOL power selection and consequently an undesirable refractive outcome.

In this study, visual and refractive outcomes after cataract surgery with the implantation (EyeCeeOne Preloaded, Bausch and Lomb, Rochester, USA) are reported. Two series of 
consecutive cataract surgeries where IOL power calculation was obtained by two different optical biometers (Aladdin, Topcon, Japan and IOL Master 500, Carl Zeiss Meditec, Germany) are presented.

\section{Methods}

We retrospectively compared two series of consecutive patients that had undergone cataract surgery in their right eye during the year 2018, followed by the implantation of the same monofocal IOL, in two different centers. In the first center (Clinica Oftalmologica Dr Parafita) optical biometry had been performed with Aladdin and in the other center (Complexo Hospitalario Universitario de Pontevedra) IOL-Master 500 had been used for optical biometry. The study adhered to the tenets of the Declaration of Helsinki, and institutional review board committee (Comite Ético da Investigación Clínica de Galicia, codigo registro 2019/494) approval was obtained.

The same experienced surgeon (PFA) performed all surgeries with the same phacoemulsification machine (Stellaris, Bausch and Lomb, Rochester, USA) and with the same surgical parameters. All the procedures were carried out under topical anesthesia with lidocaine $5 \%$, placing the main $2.2-2.5 \mathrm{~mm}$ clear corneal incision in the steeper meridian. After IOL implantation and viscoelastic aspiration, $1 \mathrm{cc}$ of intracameral cefuroxime was injected and water-tight incisions hydrated. Postoperative antibiotic and steroidal anti-inflammatory (Maxitrol, Alcon Cusi, Barcelona, Spain) eye drops were instilled 5 times daily for the first week, followed by tapered doses up to 4 weeks. The follow-up period in both groups was 6 months.

Aladdin is an optical biometer that uses optical low-coherence interferometry for axial length measurement. It also provides Placido-based corneal topography, keratometry, anterior chamber depth, central corneal thickness, white-to-white distance, aberrometry and pupillometry. IOL Master 500 is the most widely used devise for comparison and it uses partialcoherence interferometry for axial length measurement. It also provides keratometry, anterior chamber depth and white-to-white distance. SRK/T was the formula used for IOL power calculation in all cases.

EyeCeeOne Preloaded (Bausch and Lomb, Rochester, USA) is a monofocal, negative aspheric, hydrophobic intraocular lens. It has a C-loop haptic design, $360^{\circ}$ square edges, an optical diameter of $6.0 \mathrm{~mm}$ and a total diameter of $13.0 \mathrm{~mm}$. Available diopter power ranges from $+11 \mathrm{D}$ to $+27 \mathrm{D}$ in $0.5 \mathrm{D}$ steps, and in 1.0D steps from $+27 \mathrm{D}$ to $+30 \mathrm{D}$. The recommended SRK/T optical A constant is 119.7. In the present study, after optimizing the constant with the first 15 surgeries, 119.7 was used in the Aladdin group and 119.2 in the IOL-Master group. SRK/T formula was used in every case.

Exclusion criteria included corneal astigmatism greater than $1.5 \mathrm{D}$; intraoperative complications such as posterior capsular rupture or capsular dehiscence warranting capsular

Fernandez AP | Volume 2; Issue 2 (2021) | JOAR-2(2)-014 | Research Article

Citation: Fernandez AP, et al. Visual and Refractive Outcomes after Cataract Surgery with a Monofocal

Intraocular Lens Using Two Optical Biometers: IOL Master and Aladdin with EyeCeeOne Preloaded

IOL. J Ophthalmol Adv Res. 2021;2(2):1-7.

DOI: http://dx.doi.org/10.46889/JOAR.2021.2202 
tension ring implantation and postoperative complications such as corneal edema caused by endothelial pump failure or macular edema unrelated to IOL placement (i.e. macular edema, endothelial failure). Statistical analysis was made using the unpaired Student t- Test assuming heteroscedasticity after checking for normality with the Kolmogorov-Smirnov test. A p-value $<0.05$ was considered statistically significant.

\section{Results}

Baseline characteristics of the two series of patients are presented in Table 1. There were no statistically significant differences among the groups. Thirty-eight right eyes in both groups (17 males and 21 females in the Aladdin group and 16 males and 22 females in the IOL Master groups) were included. Three eyes (7.9\%) in the Aladdin group and 5 eyes $(13.2 \%)$ in the IOL-Master group presented pseudo exfoliation syndrome.

Postoperative results are shown in Table 2. Best corrected distance visual acuity was better in the Aladdin group. There were no other differences in the rest of the postoperative visual and refractive outcomes, nor in the IOL power selection (implanted) by the surgeon or the predicted spherical equivalent suggested by the biometers. None of the patients showed substantial posterior capsular opacification during the 6-month follow-up.

In the Aladdin group, $84.2 \%$ of the patients showed a SE between $\pm 0.5 \mathrm{D}$ and $94.7 \%$ of them between $\pm 1.0 \mathrm{D}$, while in the IOL-Master group $81.5 \%$ of the patients showed a SE between $\pm 0.5 \mathrm{D}$ and $94.7 \%$ of them between $\pm 1.0 \mathrm{D}$.

\begin{tabular}{|c|c|c|c|}
\hline PRE-OP & Aladdin (n= 38) & IOL-Master $(\mathbf{n}=\mathbf{3 8})$ & P-value \\
\hline Age & $73.48 \pm 11.01(48-91)$ & $74.95 \pm 9.07(57-92)$ & 0.533 \\
\hline K1 (D) & $43.80 \pm 1.40(40.58-46.21)$ & $43.70 \pm 2.04(37.54-47.47)$ & 0.815 \\
\hline K2(D) & $44.52 \pm 1.34(42.03-46.77)$ & $44.46 \pm 2.09(38.54-48.21)$ & 0.863 \\
\hline ASTIGM (D) & $0.72 \pm 0.34(0.00-1.31)$ & $0.76 \pm 0.37(0.12-1.49)$ & 0.646 \\
\hline AXL (D) & $23.36 \pm 0.89(22.13-25.71)$ & $23.35 \pm 0.88(22.13-25.34)$ & 0.929 \\
\hline IOL EMM (D) & $21.94 \pm 2.27(16.42-25.20)$ & $21.52 \pm 1.79(15.92-24.74)$ & 0.376 \\
\hline \multicolumn{2}{|r|}{ K1: flat meridian; K2: steep meridian; Astigm: astigmatism; AXL: axial length; IOL EMM: } \\
intraocular lens power for emmetropia.
\end{tabular}

Table 1: Baseline characteristics. 


\begin{tabular}{|c|c|c|c|}
\hline & Aladdin $(\mathbf{n}=\mathbf{3 8})$ & IOL-Master $(\mathbf{n}=\mathbf{3 8})$ & P-value \\
\hline IOL Implanted & $22.21 \pm 2.21(17.0-25.5)$ & $21.86 \pm 1.78(16.5-25.0)$ & 0.439 \\
\hline Diff Impl - Emm & $0.28 \pm 0.20(-0.13-0.73)$ & $0.34 \pm 0.21(-0.47-0.68)$ & 0.217 \\
\hline Predicted SE & $-0.18 \pm 0.14(-0.5-0.09)$ & $-0.22 \pm 0.14(-0.43-0.32)$ & 0.265 \\
\hline Obtained SE & $-0.21 \pm 0.34(-1.12-0.5)$ & $-0.13 \pm 0.43(-1.38-1.13)$ & 0.359 \\
\hline Diff Obt - Pred SE & $-0.02 \pm 0.34(-0.95-0.67)$ & $0.10 \pm 0.39(-1.00-1.45)$ & 0.179 \\
\hline UDVA & $0.87 \pm 0.13(0.6-1.0)$ & $0.84 \pm 0.19(0.4-1.0)$ & 0.409 \\
\hline BCDVA & $0.97 \pm 0.07(0.8-1.0)$ & $0.95 \pm 0.08(0.8-1.0)$ & 0.245 \\
\hline Sphere & $-0.07 \pm 0.32(-0.75-1.0)$ & $0.07 \pm 0.45(-1.00-1.5)$ & 0.145 \\
\hline Cylinder & $-0.30 \pm 0.35(-1.0-0)$ & $-0.38 \pm 0.55(-1.75-0)$ & 0.472 \\
\hline
\end{tabular}

Diff Impl-Emm: difference between power of the implanted IOL and the one needed for emmetropia; Diff Obt - Pred SE: difference between obtained spherical equivalent and the predicted spherical equivalent by SRK-T formula. UDVA: uncorrected distance visual acuity; BCDVA: best corrected distance visual acuity.

Table 2: IOL power selection, spherical equivalent prediction and postoperative visual and refractive outcomes.

\section{Discussion}

As far as we know, this is the third study, after the ones by Leydolt, et al., regarding visual and refractive outcomes after cataract surgery with in-the-bag EyeCeeOne Preloaded IOL $[7,8]$. It proved to be a safe and effective IOL independent of the biometer used for IOL power calculation. It achieved refractive outcomes comparable to other monofocal IOLs of similar design, with more than $80 \%$ of patients showing a SE between $\pm 0.5 \mathrm{D}$ and more than $90 \%$ of them between $\pm 1.0 \mathrm{D}[9,10]$. In this study extremely large or short eyes were not included. Further studies are needed to assess IOL predictability in that scenario. The manufacturer suggests a $2.4 \mathrm{~mm}$ incision is preferred for IOL injection, but we believe a $2.2 \mathrm{~mm}$ incision is usually enough, although a $2.4 \mathrm{~mm}$ incision might enable a more comfortable injection without the need for fixating the eye through the paracentesis or apply counter pressure.

One of the limitations of this study is that two series of patients that had undergone cataract surgery, where IOL power calculation was performed with two different biometers, are being compared. The fact that the measurements were performed in two groups of patients, using only right eyes, without basal differences between them minimizes this source of bias.

Aladdin proved to be a reliable device, comparable to IOL-Master according to the outcomes achieved in this study. Moreover, one of the advantages of Aladdin over IOL-Master is the

Fernandez AP | Volume 2; Issue 2 (2021) | JOAR-2(2)-014 | Research Article 
built-in Placido-based topography, as irregular corneas can be assessed in the same procedure. In this series of patients, Aladdin group used 119.7 and IOL-Master group used 119.2 as Aconstant for SRK/T formula. When comparing obtained and suggested spherical equivalent, a slight hyperopic shift is found in the IOL-Master group. Despite not significant, it suggests a higher A-constant could be preferred, as surgeon's tendency was to aim for a slightly myopic outcome as seen in the Aladdin group.

A recent meta-analysis suggests that optical low-coherence interferometry readings and the partial-coherence interferometry readings are equivalent [11]. The conclusions of that metaanalysis cannot be directly extrapolated to Aladdin, as it included comparative studies with Lenstar and IOL Master. Lenstar and Aladdin share the same principle, thus, it is likely to think that may not be that different. In another study comparing Aladdin and IOLMaster, only small but statistically significant differences were found in $\mathrm{K}$ readings and anterior chamber depth, suggesting that constant optimization is necessary when calculating intraocular lens power using theoretical formulas [12]. We fully agree, indeed we used a different constant in each case.

A study conducted by Sabatino, et al., found statistically significant differences in every studied parameter measured in the same patient with IOL Master and Aladdin [5]. On the other hand, as those differences were very small, they were not considered to be clinically relevant. Prior studies, like the one conducted by Mandal, et al., comparing IOL Master 500 and Aladdin, did not found statistically significant differences between the two biometers and interobserver variability was very good for each parameter [13]. Thus, both biometers seem to be valid and little differences are found between them. Nevertheless, as demonstrated in this study, optimization of the constant is recommended.

Decimal notation was preferred because 0.9 is not present in $\log$ MAR scale, and our series of patients achieved proper visual acuities that could be underestimated with logMAR scales. Both Uncorrected and Best corrected visual acuity (UCDVA and BCVA) were good in both groups.

\section{Conclusion}

Both Aladdin and IOL Master provide accurate measurements for IOL selection with satisfactory refractive outcomes. EyeCeeOne Preloaded IOL proved to be a safe and effective IOL as no IOL related problems or capsular opacifications have been reported in these two series of patients. Further studies, including long and short eyes, and with a longer follow-up period are needed to evaluate performance of the IOL and the biometers accuracy. 


\section{References}

1. Cao K, Friedman DS, Jin S, Yusufu M, Zhang J, Wang J, et al. Multifocal versus monofocal intraocular lenses for age-related cataract patients: a system review and meta-analysis based on randomized controlled trials. Surv Ophthalmol. 2019;64(5):647-58.

2. Labiris G, Toli A, Perente A, Ntonti P, Kozobolis VP. A systematic review of pseudophakic monovision for presbyopia correction. Int J Ophthalmol. 2017;10(6):992-1000.

3. Sorkin N, Rosenblatt A, Barequet IS. Predictability of biometry in patients undergoing cataract surgery. Optom Vis Sci. 2016;93:1545-51.

4. Ortiz A, Galvis V, Tello A, Viaña V, Corrales MI, Ochoa M, et al. Comparison of three optical biometers: IOLMaster 500, Lenstar LS 900 and Aladdin. Intl Ophthalmol. 2019;39(8):1809-18.

5. Sabatino F, Findl O, Maurino V. Comparative analysis of optical biometers. J Cataract Refract Surg. 2016;42:685-93.

6. Yu SS, Song H, Tang X. Repeatability of ophtha top topography and comparison with IOL-master and lenstarLS900 in cataract patients. Int J Ophthalmol. 2017;10:1703-09.

7. Leydolt C, Schartmüller D, Schwarzenbacher L, Schranz M, Schriefl S, Menapace R. Comparison of posterior capsule opacification development with 2 single-piece intraocular lens types. J Cataract and Refractive Surg. 2017;43(6):774-80.

8. Schartmüller D, Schriefl S, Schwarzenbacher L, Leydolt C, Kundi M, Pieh S, et al. Posterior capsule opacification and Nd:YAG laser rates with two hydrophobic acrylic single-piece IOLs. Eye (Lond). 2020;34(5):857-63.

9. Rodov L, Reitblat O, Levy A, Assia EI, Kleinmann G. Visual outcomes and patient satisfaction for trifocal, extended depth of focus and monofocal intraocular lenses. J Refract Surg. 2019;35(7):434-40.

10. Toygar B, Yabas Kiziloglu O, Toygar O, Hacimustafaoglu AM. Early clinical outcome with a new monofocal microincision intraocular lens. Int Ophthalmol. 2016;36:657-64.

11. Huang J, McAlinden C, Huang Y, Wen D, Savini G, Tu R, et al. Meta-analysis of optical low-coherence reflectometry versus partial coherence interferometry biometry. Sci Rep. 2017;7(1):1-0.

12. Hoffer KJ, Shammas HJ, Savini G, Huang J. Multicenter study of optical low-coherence interferometry and partial-coherence interferometry optical biometers with patients from the United States and China. J Cataract Refract Surg. 2016;42:62-7.

13. Mandal P, Berrow EJ, Naroo SA, Wolffsohn JS, Uthoff D, Holland D, et al. Validity and repeatability of the Aladdin ocular biometer. Br J Ophthalmol. 2014;98(2):256-8. 